\title{
Convalescent Plasma Therapy for Covid-19 Patients at RSUP Dr. Wahidin Sudirohusodo Makassar
}

\author{
${ }^{1}$ Faisal Muchtar, ${ }^{2}$ Rachmawati AM, ${ }^{2}$ Asvin Nurulita, ${ }^{2}$ Sri Julyani, ${ }^{2}$ Raehana Samad, ${ }^{1}$ Andi Adil, ${ }^{3}$ Irawaty \\ Djaharuddin, ${ }^{3}$ Arief Santoso, ${ }^{3}$ Nurjannah Lihawa, ${ }^{5}$ Mudji Iswanty ${ }^{4}$ Nasrum Massi, ${ }^{1}$ Syafri K Arif, ${ }^{1}$ Rezki \\ Hardiyanti
}

\author{
${ }^{1}$ Department of Anesthesiology \\ ${ }^{2}$ Department of Clinical Pathology \\ ${ }^{3}$ Department of Pulmonology \\ ${ }^{4}$ Department of Microbiology \\ ${ }^{5}$ Department of Ethics and Medicolegal \\ RSUP Dr. Wahidin Sudirohusodo Makassar, Hasanuddin University \\ DOI: 10.29322/IJSRP.11.12.2021.p12030 \\ http://dx.doi.org/10.29322/IJSRP.11.12.2021.p12030
}

\begin{abstract}
Background : The use of convalescent plasma therapy (CPT) for COVID-19 patients based on the Plasma convalescent Therapy protocol recommended by the Plasma Convalescent Therapy Team of the Indonesian Ministry of Health

Methods : Clinical trial study conducted from April - September 2020, in the group of COVID-19 patients who were treated with WHO standard therapy for COVID-19 combined with convalescent plasma administration. The results of clinical, radiological and laboratory monitoring were compared with the control group. The research consisted of 2 stages : The first stage was the search for donors: all donors were male, had no other comorbidities with Anti-SARS-CoV-2 IgG titer by ELISA method >20 COI. The second stage was to determine the recipients : adult COVID-19 patients (18-60 years) with moderate and severe criteria, according to WHO guidelines. COVID-19 patients undergo therapy in the Special Inpatient Installation (Isolation) or Intensive Care (ICU).
\end{abstract}

Results: We got 8 potential donors from 200 people who recovered from covid-19, anti-SARS-CoV2 IgG levels was 28.38+5.4 COI. In the second stage, that received convalescent plasma therapy consisted of 4 patients moderate criteria and 4 patients severe criteria. There were 2 patients with severe criteria who died after Day +7 due to renal failure and secondary infection characterized by elevated procalcitonin levels. all patients with moderate criteria recovered. Laboratory results of hematology, hemostasis, and inflammatory markers improved before $\mathrm{H}+7$, but radiological examination results improved after Day+7

\section{discussion}

In this study, it seems that the effectiveness of giving CPT to moderate-grade patients gives better results, compared to control patients and COVID-19 patients with severe criteria. it is in accordance with several clinical trials in china

\section{Conclusion}

From this study, it can be concluded that convalescent plasma therapy can be recommended, especially in moderate-grade COVID-19 patients. Further studies on a larger scale are needed regarding the effectiveness and timing of convalescent plasma administration in mild-moderate COVID-19 patients.

Keywords: Convalescent, Plasma Therapy, COVID-19 patients and Special Inpatient Installation

\section{Background}

Coronavirus disease 2019 or Covid-19 is a disease that can cause respiratory problems and pneumonia. This disease is caused by the infection with Coronavirus 2 (SARS-CoV-2) Severe Acute Respiratory Syndrome. Clinical symptoms can vary from flu-like symptoms (cough, runny nose, pharyngitis, myalgia, and headache), to severe complications (pneumonia or sepsis). On March 11, 2020, the World Health Organization (WHO) declared Covid-19 as a pandemic, after Covid-19 cases skyrocketed to 118,000 from 114 cities with more than 4000 deaths. 
ISSN 2250-3153

Just like other respiratory infections, Covid-19 spreads from human to human through droplets when a patient coughs or sneezes. The spread of aerosols was also possible after several cases were found in a closed room and exposed to the virus to one family and medical staff who occurred in a closed ward and did not have direct contact with Covid-19 patients (Marco, 2020; WHO, 2020)

Under WHO recommendations, the diagnosis of Covid-19 is based on clinical criteria and laboratory findings through the Real Time Polymerase Chain Reaction (RT-PCR) test to detect the Covid-19 antigen in swab samples taken from the upper respiratory tract, such as the oropharynx and nasopharynx, and lower respiratory tract such as sputum, endotracheal aspiration or from bronchoalveolar lavage.

The incubation period or the period of entry of the virus until the onset of symptoms occurs on days 0-14, at this time viral antigens can be detected by the RT-PCR test, which is per WHO recommendations for laboratory tests to detect Covid-19 antigens. As an immunological response to the COVID-19 virus antigen, anti-SARS-CoV-2 immunoglobulin-M (IgM) formed on day 7 and peaked on day 14 , then slowly decreased and disappeared on day 21 . Immunoglobulin-G (IgG) anti-SARS-CoV-2 will form on the 14th day and then increase in the blood for a long time, until now there has been no scientific explanation about when IgG anti-SARS-CoV-2 disappears in human blood. (Liu L. 2002)

The recovery period is the time when the $\operatorname{IgM}$ response has disappeared and the $\operatorname{IgG}$ response has been established. Rapid IgM and $\mathrm{IgG}$ tests can be performed to determine whether the patient is recovering, IgM will give a negative result and $\operatorname{IgG}$ will be reactive. During the recovery period, anti-SARS-CoV-2 IgG is available in the patient's blood. To determine the level of IgG in plasma, it can be done by using the Enzyme Link Immunoassay (ELISA), Electro-chemiluminiscen Immunoassay (ECLIA) and Neutralizing antibody methods.

The use of convalescent plasma or plasma of patients who recovered from COVID-19 may be considered for COVID-19 patients. The use of the patient's blood plasma during the recovery period in viral infections with clinical manifestations of Severe Acute Respiratory Syndrome (SARS) has been used in previous cases such as the Middle East Respiratory Coronavirus Syndrome (MERS$\mathrm{CoV}$ ) case in 2012, the SARS coronavirus (SARS) case - CoV), Spanish influenza A (H1N1), and avian influenza A (H5N1) in 2009. Moreover, WHO developed Interim Guidance for National Health Authorities and Blood Transfusion Services for the Conventional Use of Whole Blood or Plasma Collected from Patients Recovering from Disease Ebola Virus for Transfusion, as an Empirical Treatment during the Outbreak in September 2014.

Based on this, we from RSUP Dr. Wahidin Sudirohusodo Makassar in collaboration with Research and Technology Brin and LPDP conducted a trial of using Convalescent Plasma in moderate and severe COVID-19 patients. The research team came from the departments of Anesthesiology, Pulmonology, Clinical Pathology, Ethics and Medicolegal. This research obtained permission and ethics from Hasanuddin University Medicolegal and National Technology Research. The specific purpose of the study was to determine differences in clinical conditions based on the sofa score of Covid-19 patients before and after convalescent plasma transfusion. To know the difference in the results of laboratory parameters (neutrophil lymphocyte ratio, C-Reactive Protein, Interleukin-6, D. Dimer) before and after convalescent plasma transfusion. to know the differences in laboratory test results and clinical conditions of Covid-19 patients with the control group.

Research method: Clinical trial study conducted from April - September 2020, in the group of COVID-19 patients who were treated with WHO standard therapy for COVID-19 combined with convalescent plasma administration. The results of clinical, radiological and laboratory monitoring were compared with the control group.

The research consisted of 2 stages.

The first stage was the search for donors: The donor criteria were the same as the criteria for apheresis donors according to the criteria of the Minister of Health regulation 91 of 2015, with the results of the SARS-CoV-2 RT PCR confirmed negative 2 times in a row. All donors were male, had no other comorbidities: Diabetes Mellitus on insulin therapy, severe hypertension, CKD. Anti-SARS-CoV$2 \mathrm{IgG}$ titer by ELISA method $>20$ COI. Donor screening examination was a regular blood check, with a hematology analyzer sysmex XN 1000, blood type examination using the Gel Test method.

The second stage was to determine the recipients, which were adult COVID-19 patients (18-60 years) with moderate and severe criteria, according to WHO guidelines. COVID-19 patients undergoing therapy in the Special Inpatient Installation (Isolation) or Intensive Care (ICU) at the Hospital.

Criteria for Moderate COVID-19 (Burhan et al., 2020; WHO, 2020a):

- Signs and symptoms associated with pneumonia are found

- Does not require oxygen supplementation therapy

- Does not meet any of the criteria for severe pneumonia

Criteria for Severity of COVID-19 (Wu and McGoogan, 2020)

This publication is licensed under Creative Commons Attribution CC BY.

http://dx.doi.org/10.29322/IJSRP.11.12.2021.p12030

www.ijsrp.org 
- Shortness of breath with respiration rate 30 times per minute

- Oxygen saturation (SpO 2) $93 \%$ in room air

- $\mathrm{PaO} 2$ / FiO 2 ratio $300 \mathrm{mmHg}$

- Chest $\mathrm{x}$-ray lesion worsens $>50 \%$ in $24-48$ hours

The control group was COVID-19 patients who were randomly selected and matched by sex, age group and moderate or severe severity, who received WHO standard Covid-19 standard therapy.

Convalescent plasma was transfused at H0, given 400cc in divided doses of 200cc each per 24 hours.

In the convalescent plasma therapy group, clinical, radiological and laboratory monitoring was carried out according to the following protocol:

Table 1. Protocols for monitoring convalescent plasma therapy in COVID-19 patients

\begin{tabular}{|c|c|c|c|c|c|c|c|}
\hline \multirow[b]{2}{*}{ Activity } & \multicolumn{7}{|c|}{ Monitoring day } \\
\hline & -3 & -1 & 0 & +1 & +2 & +7 & $\begin{array}{l}+28 \text { and/or } \\
\text { discharge }\end{array}$ \\
\hline Standard Treatment & $\sqrt{ }$ & $\sqrt{ }$ & $\sqrt{ }$ & $\sqrt{ }$ & $\sqrt{ }$ & $\sqrt{ }$ & $\sqrt{ }$ \\
\hline Informed consent & $\sqrt{ }$ & & & & & & \\
\hline Screening eligibility & $\sqrt{ }$ & & & & & & \\
\hline Transfusion plasma convalescent & & & $\sqrt{ }$ & & & & \\
\hline Clearance viral examination & & $\sqrt{ }$ & & $\sqrt{ }$ & $\sqrt{ }$ & $\sqrt{ }$ & \\
\hline chest $\mathrm{x}$-ray examination & & $\sqrt{ }$ & & $\sqrt{ }$ & $\sqrt{ }$ & $\sqrt{ }$ & \\
\hline WBC and Diff count examination & & $\sqrt{ }$ & & $\sqrt{ }$ & $\sqrt{ }$ & $\sqrt{ }$ & \\
\hline C-Reactive Protein, Procalcitonin, IL-6 Examination & & $\sqrt{ }$ & & $\sqrt{ }$ & $\sqrt{ }$ & $\sqrt{ }$ & \\
\hline Liver Function (ALT, AST) & & $\sqrt{ }$ & & $\sqrt{ }$ & $\sqrt{ }$ & $\sqrt{ }$ & \\
\hline Renal Function Examination (Urea, creatinine) & & $\sqrt{ }$ & & $\sqrt{ }$ & $\sqrt{ }$ & $\sqrt{ }$ & \\
\hline D- Dimer Examination & & $\sqrt{ }$ & & $\sqrt{ }$ & $\sqrt{ }$ & $\sqrt{ }$ & \\
\hline Peripheral blood smear & & $\sqrt{ }$ & & $\sqrt{ }$ & $\sqrt{ }$ & $\sqrt{ }$ & \\
\hline clinical parameter examination & & $\sqrt{ }$ & & $\sqrt{ }$ & $\sqrt{ }$ & $\sqrt{ }$ & $\sqrt{ }$ \\
\hline Sofa score (only for critically ill) & & & & $\sqrt{ }$ & $\sqrt{ }$ & $\sqrt{ }$ & $\sqrt{ }$ \\
\hline Monitoring AE and SAE & & & & $\sqrt{ }$ & $\sqrt{ }$ & $\sqrt{ }$ & $\sqrt{ }$ \\
\hline
\end{tabular}

\section{Results :}

In the first stage where convalescent plasma donor screening was carried out at Dr. Wahidin Sudirohusodo, 24 potential donors met the criteria for apheresis donors from 200 COVID-19 survivors.

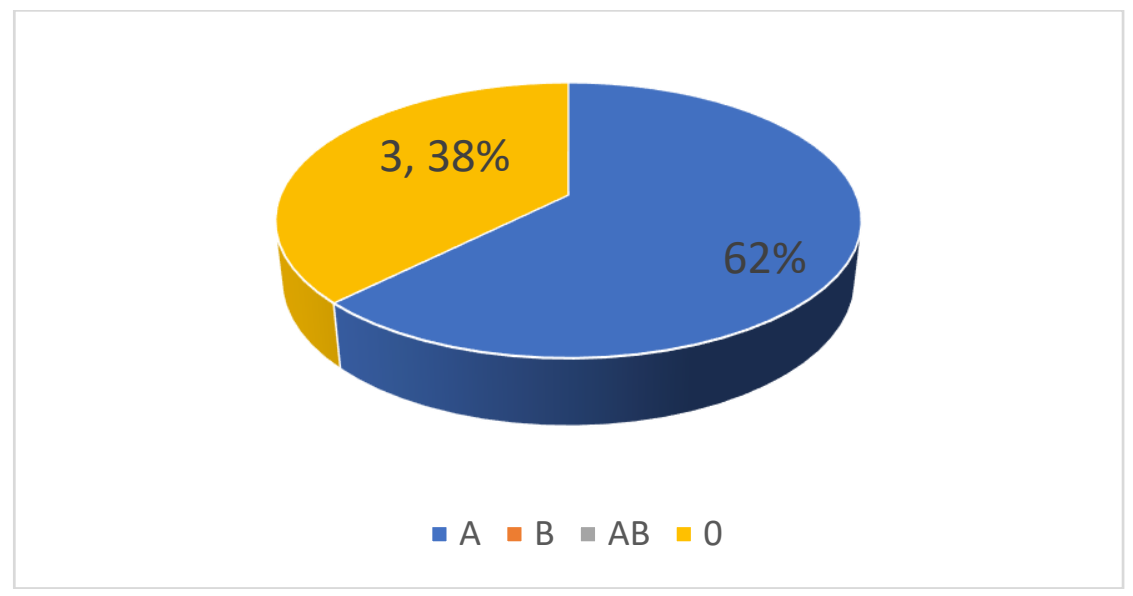

Figure 1. Distribution of convalescent plasma donor blood groups 
Anti-SARS-CoV-2 IgG antibody levels were examined and 8 donors who met the inclusion criteria were netted, with clinical symptoms when infected with SARS-CoV2 varied, ranging from coughing symptoms resembling flu symptoms to severe dry cough and shortness of breath. Of the 8 potential convalescent plasma donors, $62 \%$ had blood type A and $3.38 \%$ had blood type O, no blood type $\mathrm{B}$ and $\mathrm{AB}$ donors were found.

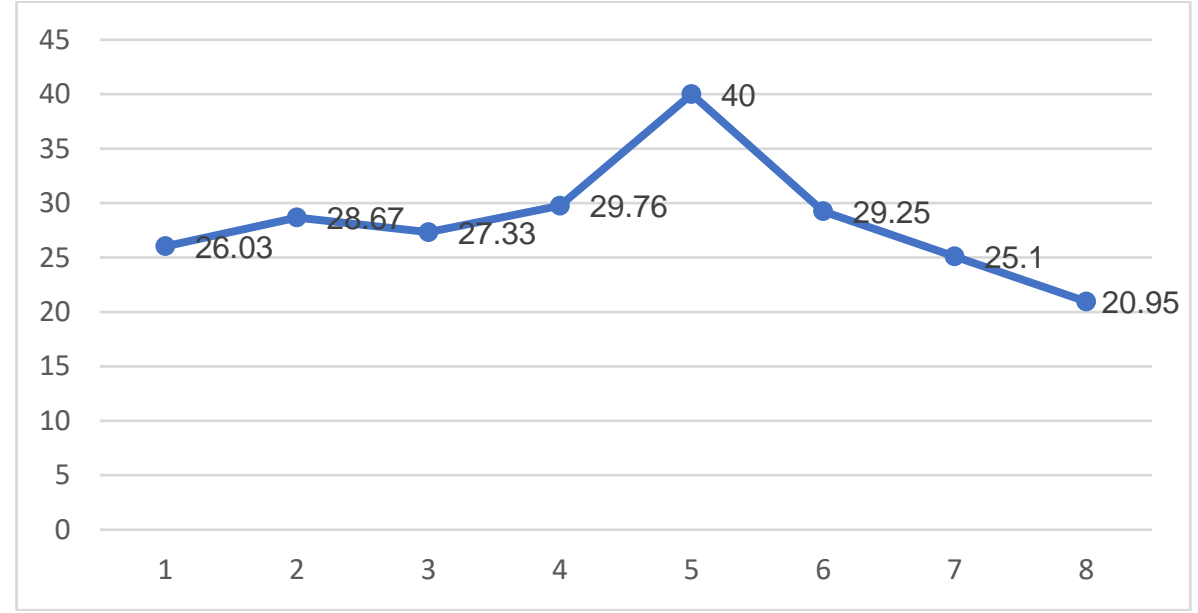

Figure 2. Distribution of anti-SARS-CoV2 IgG levels in convalescent plasma donors

The mean \pm standard deviation of anti-SARS-CoV2 IgG levels in 8 convalescent donors was $28.38+5.4$ COI. The convalescent plasma obtained was stored at $-20 \mathrm{oC}$.

In the second stage, clinicians who treat COVID-19 patients determined patients who met the criteria as recipients with moderate and severe COVID-19 criteria then determined the control group. The group that received convalescent plasma therapy consisted of 4 patients with moderate criteria and 4 patients with severe criteria. Similarly, the control group consisted of 4 patients with moderate criteria and 4 patients with severe criteria.

In general, 4 moderate COVID-19 patients who received convalescent plasma therapy recovered $100 \%$. Of the 4 severe grade patients with the same treatment, only $50 \%$ recovered, 2 severe grade patients died by Day+7 due to secondary infection characterized by elevated procalcitonin levels

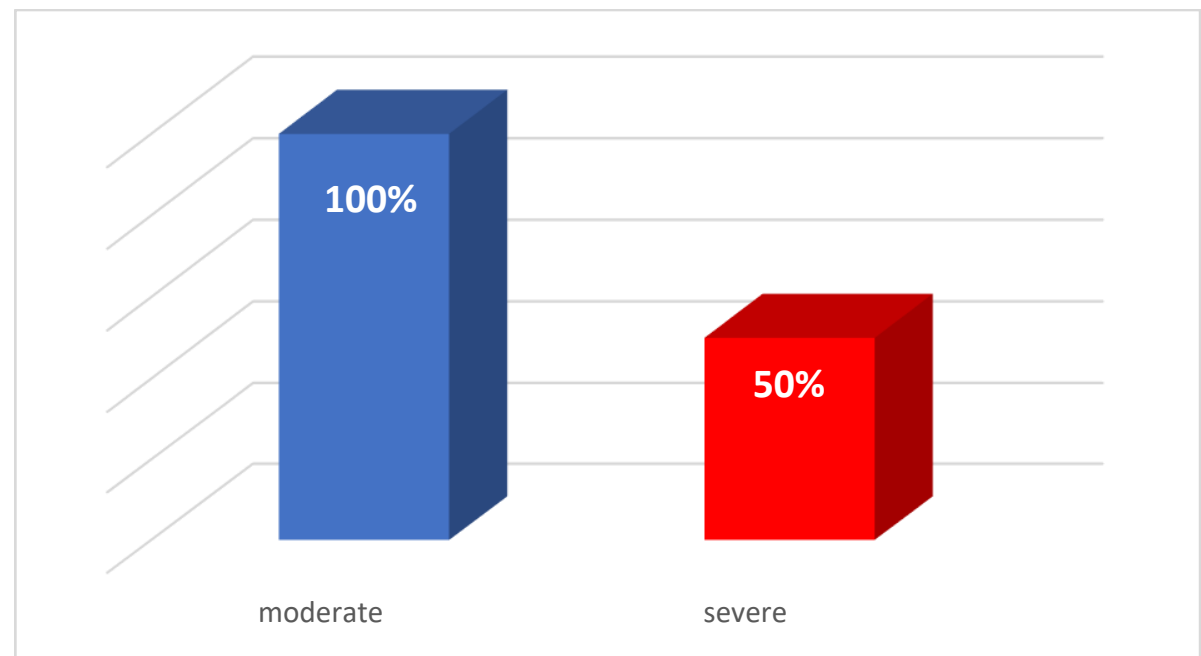

Figure 3. The cure rate of convalescent plasma therapy in moderate and severe COVID-19 patients receiving convalescent plasma therapy

Based on clinical and laboratory monitoring, the results are as shown in Table 1.

Table 1. Clinical monitoring and average laboratory results of moderate-grade COVID-19 patients Get Convalescent Plasma Therapy 


\begin{tabular}{|c|c|c|c|c|c|c|}
\hline \multirow[t]{2}{*}{ no } & \multirow[t]{2}{*}{ Laboratory parameters } & \multicolumn{5}{|c|}{ Monitoring day } \\
\hline & & Day-1 & Day+1 & Day +2 & Day +7 & Day+28 \\
\hline 1 & $\begin{array}{l}\text { Neutrophil lymphocyte } \\
\text { (NLR) }\end{array}$ & 8.2 & 4.8 & 6.6 & 4.2 & 2.3 \\
\hline 2 & D. Dimer & 2.8 & 7.8 & 4.2 & 4.23 & $*$ \\
\hline 3 & Interleukin-6 & 54.5 & 73.3 & 64.5 & 53.3 & $*$ \\
\hline 4 & C- Reactive Protein & 91.9 & 101.6 & 35.2 & 50.6 & $*$ \\
\hline 4 & Creatinine & 0.7 & 0.9 & 0.8 & 0.8 & 0.73 \\
\hline 5 & Urea & 37 & 50 & 60.2 & 53 & 66.5 \\
\hline 6 & SGOT & 69 & 50 & 40 & 41 & 33 \\
\hline 7 & SGPT & 81 & 78 & 58 & 79 & 53 \\
\hline 8 & Procalcitonin & 0.05 & 0.05 & 0.05 & 0.05 & 0.05 \\
\hline 9 & SOFA Score & 2 & 2 & 0 & 0 & 0 \\
\hline
\end{tabular}

Table 1 shows the average laboratory results of moderate-grade COVID-19 patients receiving convalescent plasma therapy. As $100 \%$ of patients recovered after Day+7, further clinical and laboratory monitoring was performed at outpatient visits.

Table 2. Clinical monitoring and mean laboratory results of severe COVID-19 patients

Get Convalescent Plasma Therapy

\begin{tabular}{lllllll} 
no & Laboratory parameters & \multicolumn{5}{c}{ Monitoring day } \\
\cline { 3 - 7 } & & Day-1 & H+1 & H+2 & Day-1 & H+28 \\
\hline 1 & Neutrophil lymphocyte Ratio (NLR) & 11.2 & 13.1 & 18.1 & 14.6 & 4.2 \\
\hline 2 & D. Dimer & 19.8 & 20.7 & 29.1 & 20.9 & 4.23 \\
\hline 3 & Interleukin-6 & 133.8 & 166.7 & 178 & 166.6 & $*$ \\
\hline 4 & C- Reactive Protein & 211.7 & 176.8 & 133.3 & 87.9 & 62.3 \\
\hline 4 & Creatinine & 1.23 & 1.05 & 1.22 & 2.22 & 0.85 \\
\hline 5 & Urea & 45 & 56 & 56.1 & 53 & 46 \\
\hline 6 & SGOT & 101.5 & 91.7 & 84.2 & 70.2 & 33.5 \\
\hline 7 & SGPT & 95 & 66.7 & 75.9 & 51 & 28.5 \\
\hline 8 & Procalcitonin & 3.85 & 6.19 & 1.92 & 9.99 & 0.3 \\
\hline 9 & SOFA Score & 4 & 4 & 4 & 4 & 1 \\
\hline
\end{tabular}

Clinical and laboratory monitoring of severe COVID-19 patients was carried out in the intensive care unit until Day+28 administration of convalescent plasma. Two patients died on Day+7 and 2 other patients recovered.

Furthermore, laboratory results were compared between moderate-grade COVID-19 patients and moderate-grade controls. 


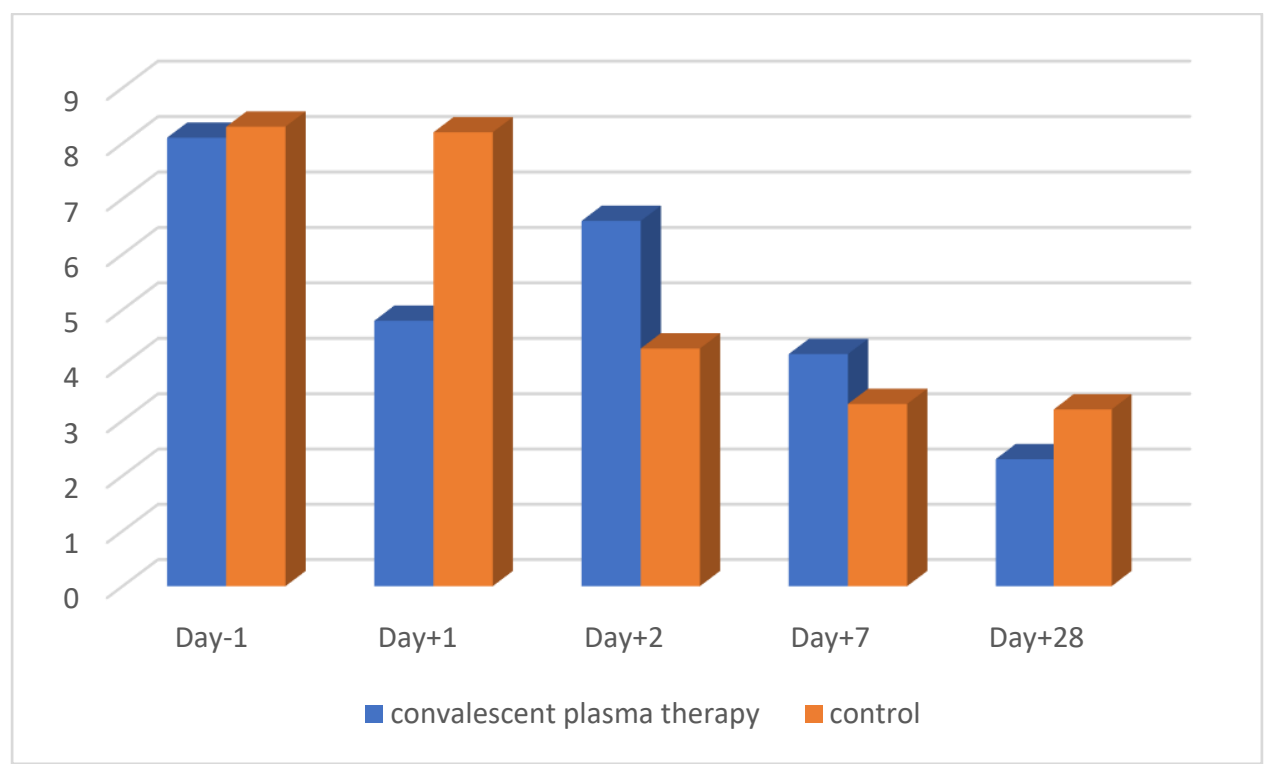

Figure 4. Comparison of NLR in moderate COVID-19 patients

Figure 4 shows the comparison of NLR between groups of moderate COVID-19 patients. On Day+1 there was a decrease in the convalescent plasma therapy group, but from Day+2 to Day+7 the NLR was almost the same in both groups, but on Day+28 the NLR in the convalescent plasma therapy group decreased compared to the control group.

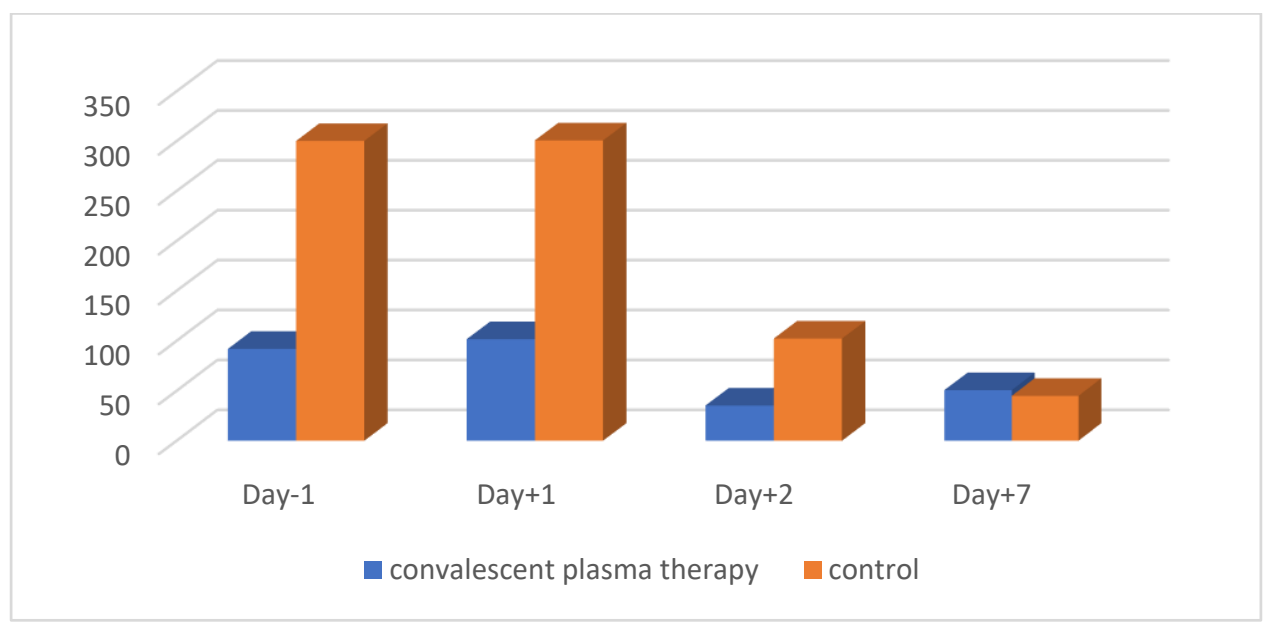

Figure 5. Comparison of CRP levels in moderate COVID-19 patients.

Figure 5 shows CRP levels in moderate COVID-19 patients. On Day+1, there was an increase in the convalescent plasma therapy group, but from Day+2 to day +7 , the decrease was almost the same as in the control group. 


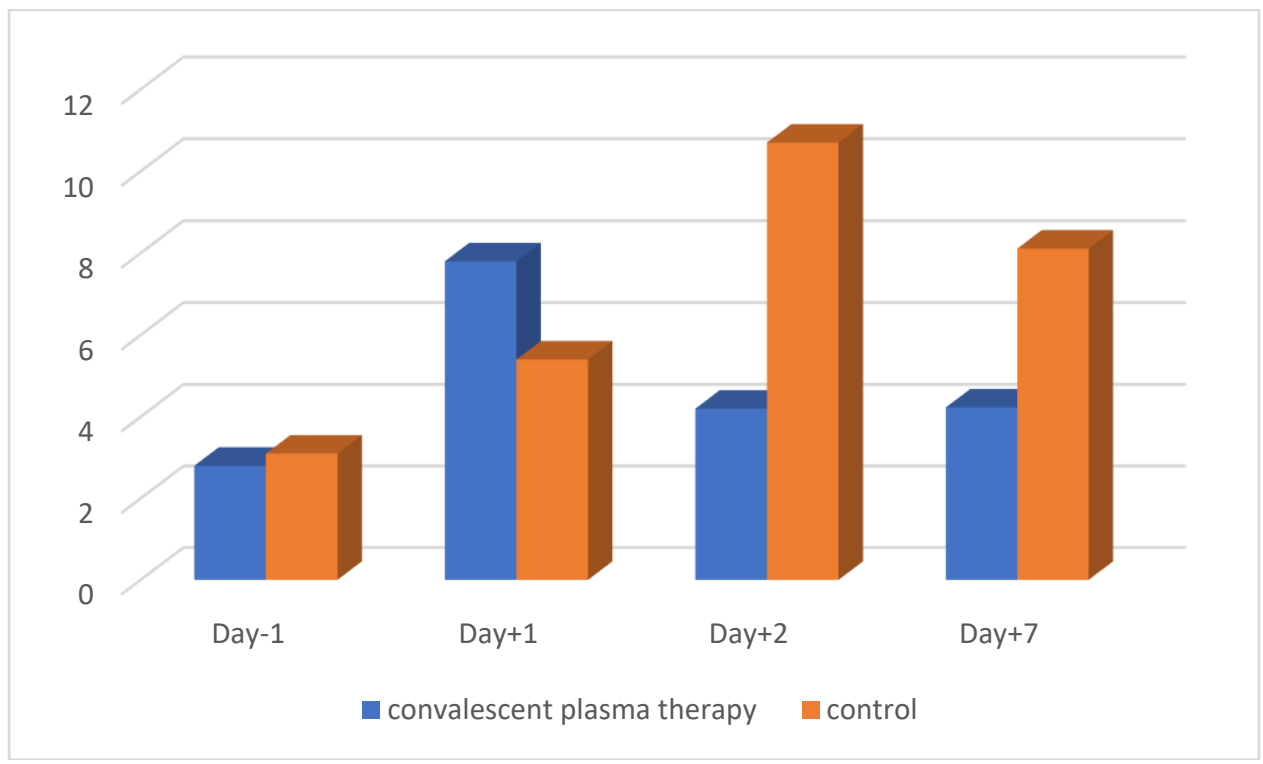

Figure 6. Comparison of D-Dimer levels in moderate COVID-19 patients

Figure 6 shows the comparison of D-Dimer levels between the group of moderate COVID-19 patients and the control group. On Day +1 , there was an increase in both the convalescent plasma therapy group and the control group, but from Day +2 to Day +7 there was a decrease in the convalescent plasma therapy group, while the control group saw an increase.

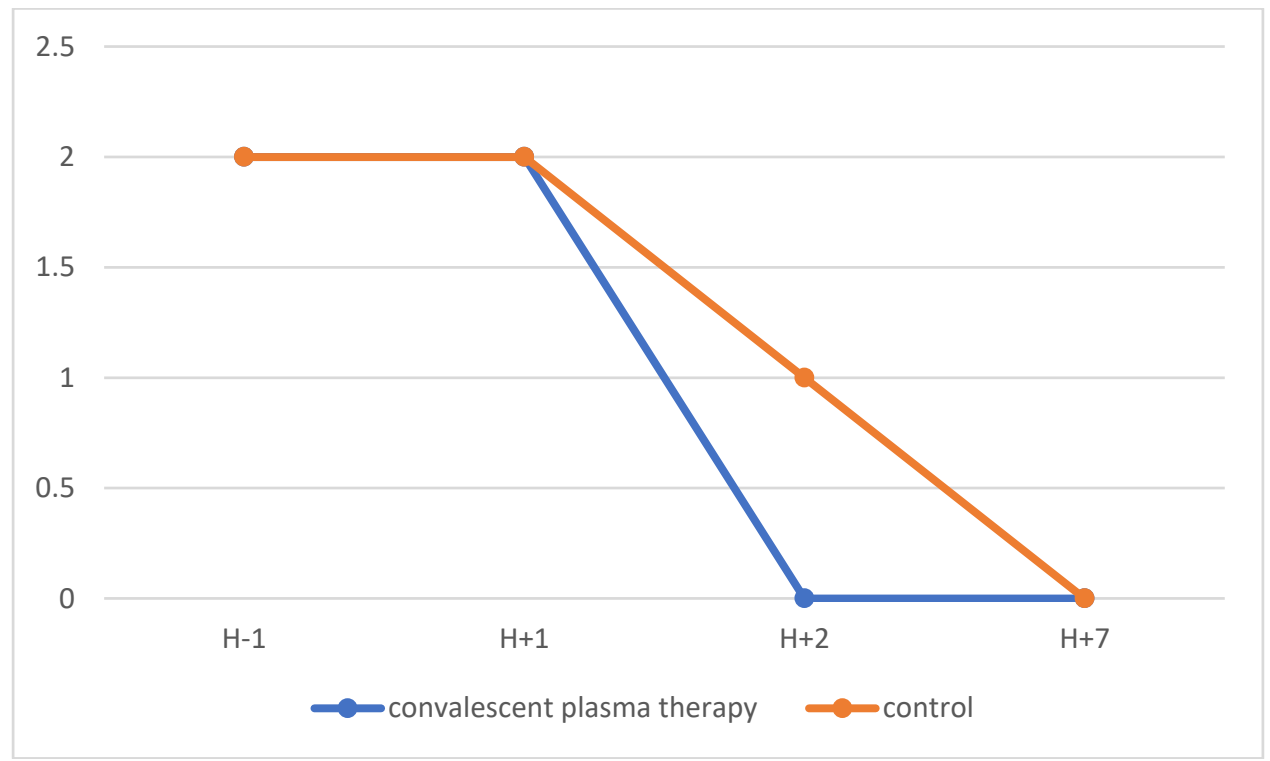

Figure 7. Comparison of SOFA scores in moderate COVID-19 patients

Figure 7 shows a comparison of SOFA scores in moderate COVID-19 patients. From Day-1 to Day+7 there was no change in both groups.

Furthermore, laboratory results were compared between severe COVID-19 patients. 


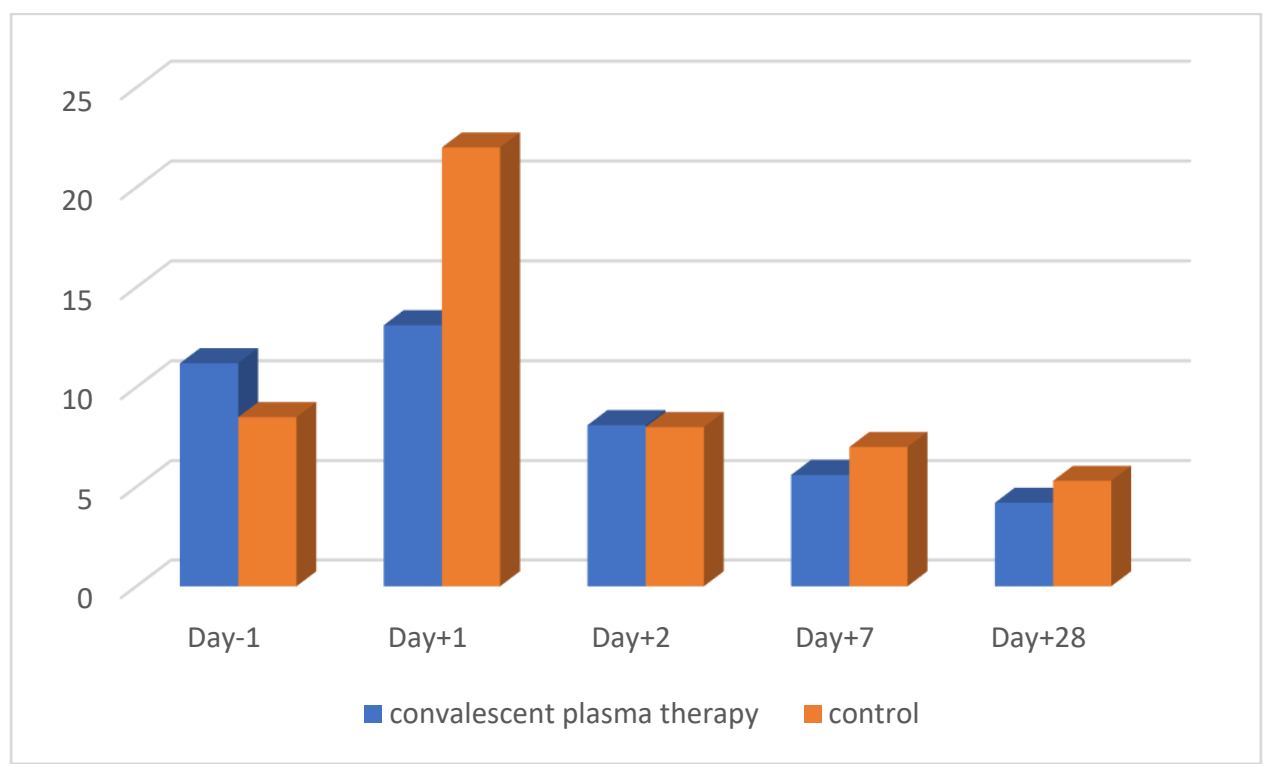

Figure 8. Comparison of NLR in severe COVID-19 patients

Figure 8 shows the comparison of NLR between groups of severe COVID-19 patients. On Day+1, there was an increase in both groups and from Day+2 to Day+7, there was almost the same decrease in both groups.

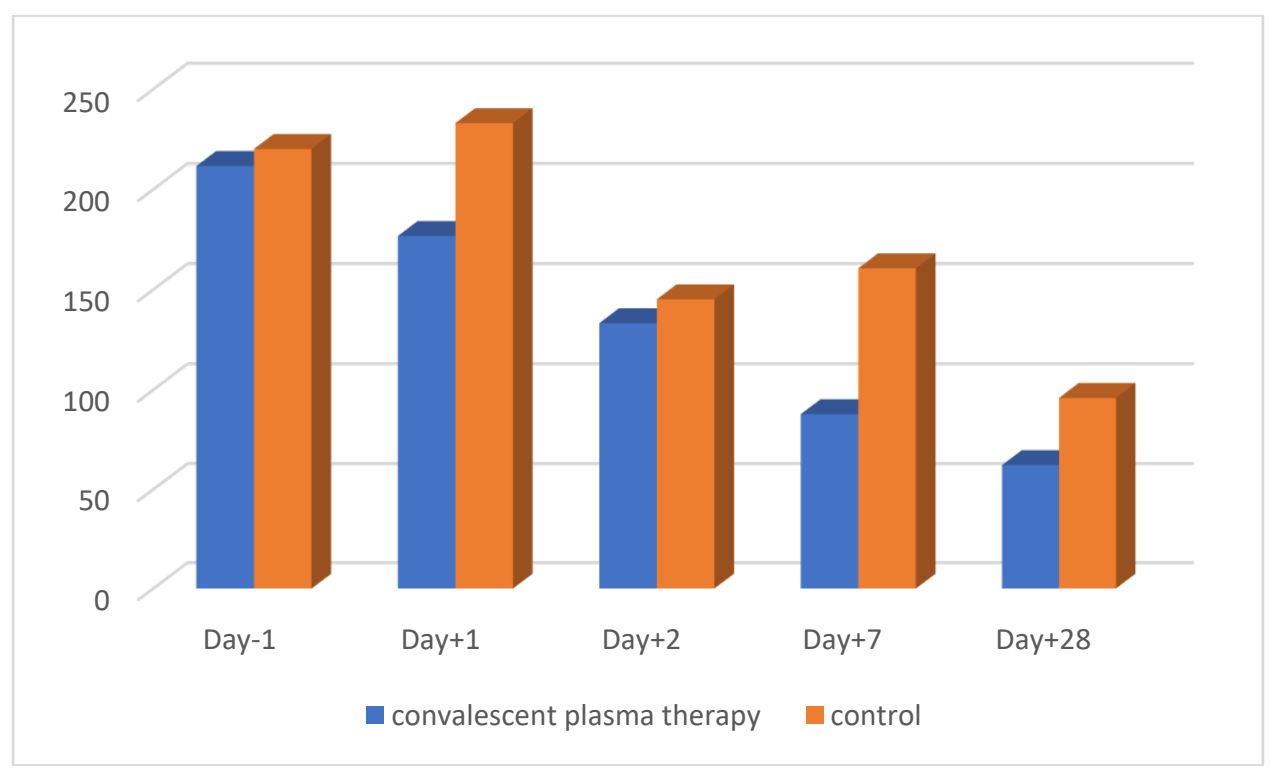

Figure 9. Comparison of CRP levels in severe COVID-19 patients

Figure 9 shows a comparison of CRP levels in severe COVID-19 patients. From Day+1 to Day+7, there was a decrease in the convalescent plasma therapy group, while in the control group there was an increase on Day +1 , a decrease on Day +2 , an increase on Day+7, and a decrease again on Day+28. 


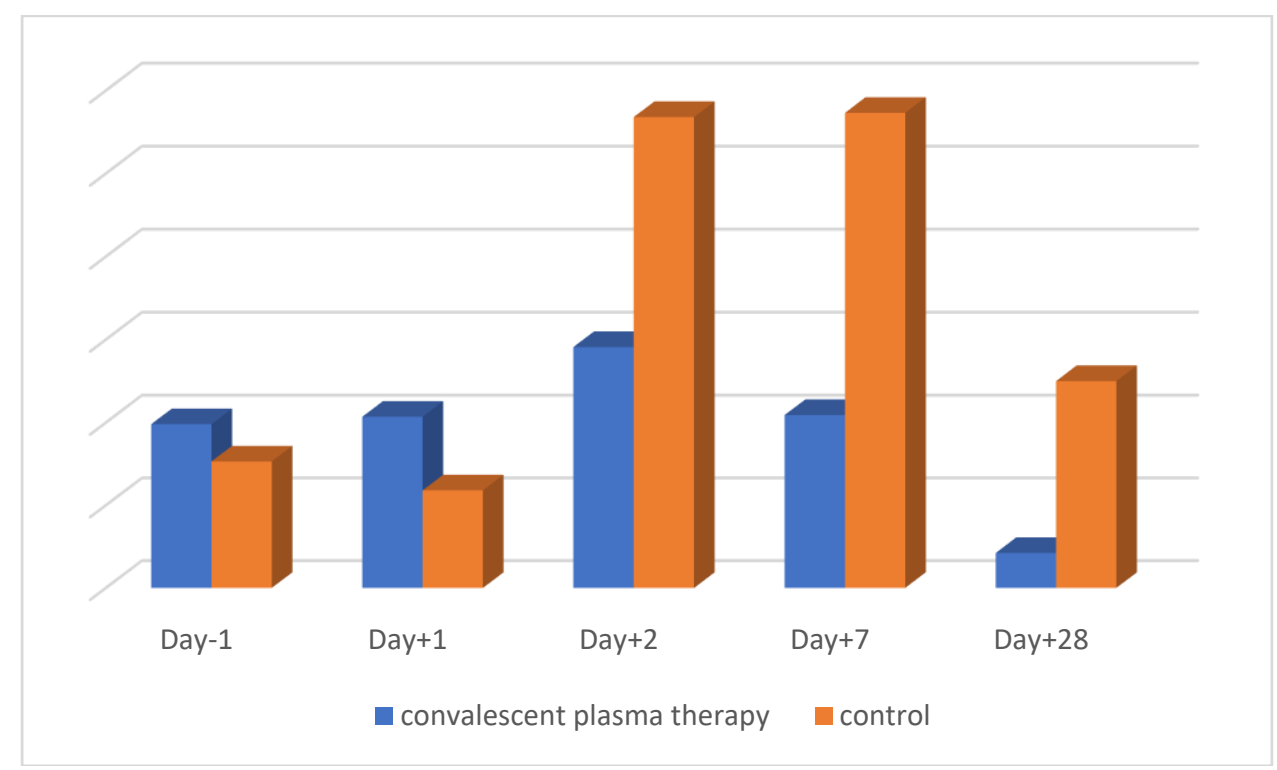

Figure 10. Comparison of D-Dimer levels in severe COVID-19 patients

Figure 10 shows a comparison of D-Dimer levels in severe COVID-19 patients which increased on Day+1 and Day +2 , but appeared to decrease on Day+7 and Day+28 in the convalescent plasma therapy group, while in the control group decreased on Day +1 and increases on Day+2, then decreases on Day+28.

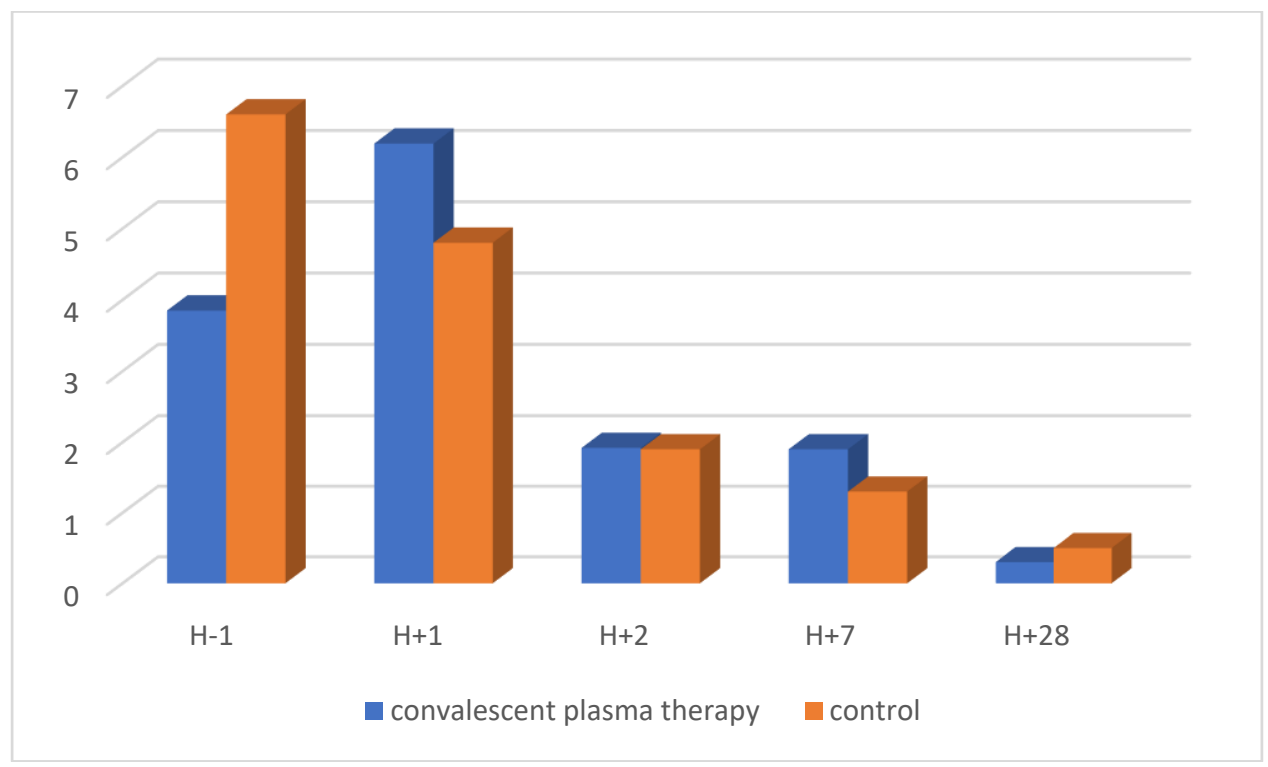

Figure 11. Comparison of Procalcitonin levels in severe COVID-19 patients

Figure 11 shows a comparison of procalcitonin levels in the severe COVID-19 patient group. On Day+1, there was an increase in procalcitonin levels in both groups and from Day+2 to Day +28 , there was a decrease in procalcitonin levels in both groups. 


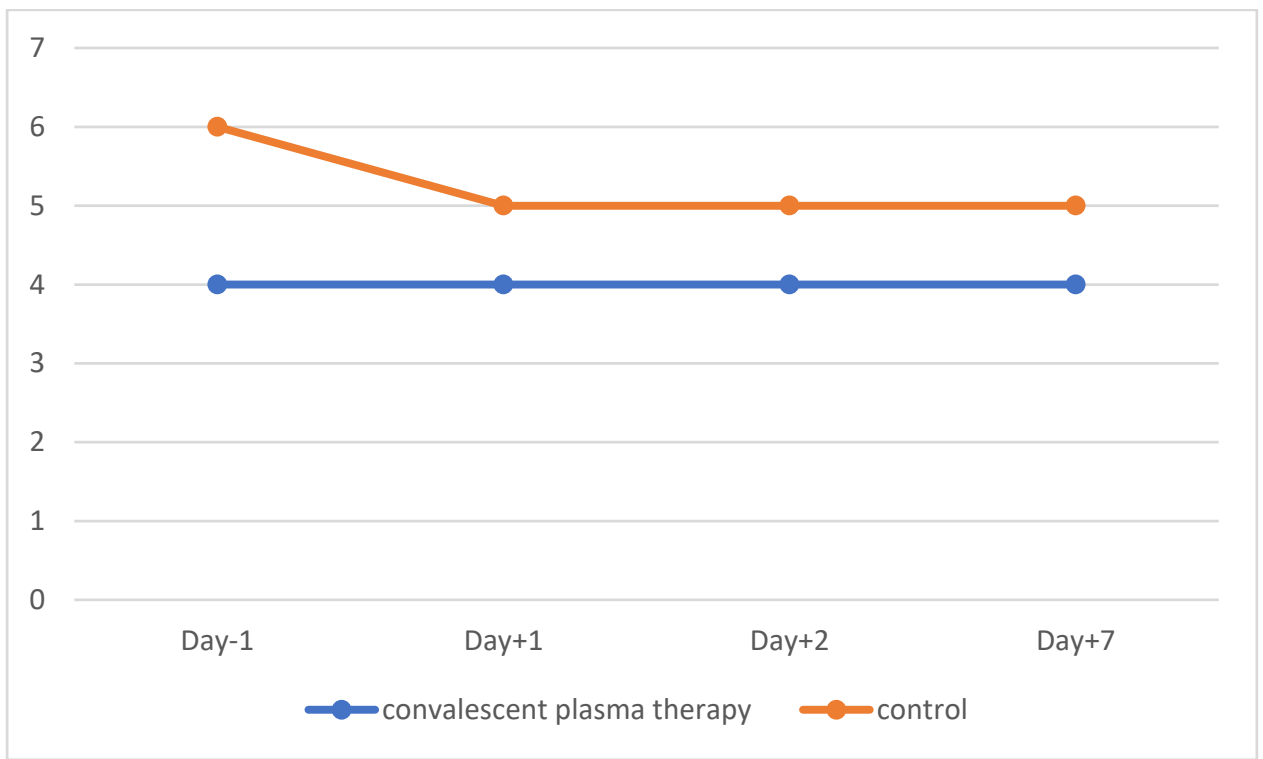

Figure 12. Comparison of SOFA scores in severe COVID-19 patients

Figure 12 shows a comparison of SOFA scores in severe COVID-19 patients. The convalescent plasma therapy group looked the same from Day+1 to Day+7, whereas in the control group there was a decrease on Day+1 but there was no change from Day+2 to Day+7.

\section{Discussion}

Convalescent plasma therapy is based on passive immunization by utilizing antibodies formed in patients with COVID-19. These antibodies are formed by humoral immune cells and are present in the plasma of patients with COVID-19. When someone is attacked by COVID-19, two types of antibodies arise in the body, namely antibodies or immunoglobulin M (IgM) and immunoglobulin G (IgG). Data from valid articles regarding COVID-19 states that IgM will appear from the first day of being exposed to COVID-19 and reach its peak on days 7-14, then it will decrease and disappear in about a month. Meanwhile, IgG will appear about a week after IgM appears and will reach its peak on days 21-28. After that, it will last for 4 months, then it will decrease gradually until it disappears in 36 months.

The immediate use of convalescent plasma holds promise when vaccines and specific therapies are being evaluated. Convalescent plasma from donors recovering from COVID-19 can be very promising when used prophylactically or examined soon after disease onset (within 14 days). Protection can last from several weeks to several months (Brown et al, 2020). A study in Wuhan, China, regarding convalescent plasma therapy for COVID-19 patients showed that convalescent plasma therapy was effective and specific for COVID-19. This intervention is of particular significance for the elimination of SARS-CoV-2 and is believed to be a promising therapy during the COVID-19 pandemic crisis (Ye et al, 2020).

In the early stages of this study, only convalescent plasma donors with blood groups $\mathrm{A}$ and $\mathrm{O}$ were found so that patients who were included in the convalescent plasma therapy group to receive immediate therapy were only blood types A and O. Mean \pm standard deviation of anti-SARS-CoV2 IgG levels at 8 the convalescent donor was $28.38+5.4$ COI. A study that supports the benefits of convalescent plasma for the treatment of COVID-19 at various stages of disease emphasizes the importance of measuring antibody levels in convalescent plasma before transfusion to ensure adequate antibody levels (Maor et al, 2020). Another study found that most COVID-19 patients had high neutralizing antibody titers at the time of hospital admission. Antibody screening and prioritizing convalescent plasma for at-risk groups with new onset of symptoms will be key to identifying patients who benefit from convalescent plasma (Gharbharan et al, 2020).

A total of 16 patients were included in this study. Of the 16 patients, 8 patients were included in the convalescent plasma therapy group and 8 others were included in the control group. In each group, there were 4 moderate COVID-19 patients and 4 severe COVID-19 patients. In the convalescent plasma therapy group, patients with moderate-grade COVID-19, all patients were declared cured while in patients with severe degrees only 50\% recovered. This is consistent with a study from Indonesia regarding convalescent plasma therapy in moderate to severe COVID-19 patients which emphasized the safety of convalescent therapy. Although a trend of improvement was found in patients with moderate disease, it cannot be concluded whether this outcome was due to convalescent plasma therapy alone (Rejeki et al, 2021). 
Similar results were obtained in a multicenter interventional study in Kuwait on convalescent plasma therapy in cases of moderate and severe SARS-CoV-2 infection which showed that convalescent plasma administration was significantly associated with improved clinical outcomes. The 30-day survival increased significantly in the moderate case group (Alsharidah et al, 2021).

A total of 2 patients with severe degrees recovered after administration of convalescent plasma therapy. These findings are consistent with studies examining the effectiveness of convalescent plasma in patients with severe COVID-19 showing that convalescent plasma therapy is well tolerated and can potentially improve clinical outcomes by neutralizing viremia in severe COVID-19 cases. The optimal dose and timing of administration and the benefits of convalescent plasma therapy require further studies in large-scale controlled studies (Duan et al, 2020). Research in East Anatolia on the life-saving effects of convalescent plasma therapy suggests that convalescent plasma provides a promising urgent therapeutic option while existing drugs are still being evaluated and new specific vaccines are still being developed. In this study, convalescent plasma appears to be effective in severe and critical COVID-19 patients and can reduce ICU admission rates. It was found that all patients with early-stage COVID-19 not requiring mechanical ventilation improved with convalescent plasma therapy. However, despite convalescent plasma therapy, 6 of the 17 patients requiring mechanical ventilation died. In early-stage COVID-19 patients who do not require mechanical ventilation, convalescent plasma therapy can be used as a curative therapy option (Erkurt et al, 2020).

In contrast to these findings, a study in patients with severe pneumonia due to COVID-19 showed that convalescent plasma therapy did not reduce mortality or improve other clinical outcomes at day 30 (Simonovich et al, 2020). Another study showed that there were no significant differences in primary or secondary outcome parameters between convalescent plasma therapy and standard therapy, although the number of patients requiring ventilation (non-invasive or invasive) was lower and the period was shorter in patients given convalescent plasma. However, the study also demonstrated that convalescent plasma therapy appears to be safe in COVID-19 patients treated with hypoxia (AlQahtani et al, 2021).

The mechanism of action of IgG anti-SARS-CoV-2 is to neutralize the virus so that it is very effective in mild and moderate COVID19 patients and less effective in severe and critical degrees with multiple organ disorders. This explains the basic workings of convalescent plasma therapy, namely by giving plasma from COVID-19 patients who have recovered to COVID-19 patients who are still suffering from the disease. Currently, the administration of convalescent plasma therapy is still focused primarily as a therapy for patients with COVID-19. If this gives satisfactory results, it is not impossible that it will be applied as protection for groups who are vulnerable to COVID-19, such as medical personnel who are in direct contact with COVID-19 patients or certain comorbid groups as written by Arturo Casadevall, one of the pioneers of convalescent plasma therapy in the US. In addition, various studies from China, Korea, Iran, and other countries in the world currently show that convalescent plasma therapy gives very promising results. Research studies in China, which was the first epicenter of the Covid-19 explosion in Wuhan, showed that administering convalescent plasma therapy to 10 COVID-19 patients provided healing for all patients (Duan K, et al 2020) and administration of TPK to five critical patients also provided healing. for these five patients (Guan S, et al 2020).

Inflammatory markers examined in this study consisted of NLR (neutrophile-to-lymphocyte ratio), CRP levels, and D-Dimer levels. In patients with moderate COVID-19, the NLR ratio in the convalescent plasma therapy group was almost the same as in the control group but decreased on day 28. The comparison of CRP levels was almost the same in both groups, while the comparison of D-Dimer levels showed a decrease in the convalescent plasma therapy group. In patients with severe COVID-19, the NLR ratio was almost the same between the two groups, CRP levels showed a decrease in the convalescent plasma therapy group, and changes in D-Dimer levels were almost the same between the two groups.

A study also demonstrated lower CRP levels in the convalescent plasma therapy group in moderate and severe COVID-19 patients (Gharbharan et al, 2020; Alsharidah et al, 2021). In another study, CRP levels at the time of discharge from the hospital showed a significant difference where higher CRP levels were found in the convalescent plasma therapy group (AlQahtani et al, 2021). DDimer levels in several studies did not show significant differences (AlQahtani et al, 2021; Alsharidah et al, 2021).

In contrast to a randomized controlled trial of convalescent plasma for severe and critically ill COVID-19 patients, which showed that inflammatory markers consisting of D-dimer, CRP, and IL-6 did not show significant differences at days 3, 7, and 14. after administration of convalescent plasma. In that study convalescent plasma added to standard steroid therapy did not result in a higher proportion of clinical improvement at day 28 (Sekine et al, 2021).

This study has several limitations. First, the research was conducted from April to September 2020 at Dr. RSUP. Wahidin Sudirohusodo encountered many obstacles in the early stages of finding a donor. In addition to COVID-19 survivors who are still in the healing phase, there is also a lack of understanding of convalescent plasma, so most of the 200 survivors refuse to donate plasma. Second, the number of eligible plasma donors was only 8 out of 24 survivors who underwent screening so that the sample size was small. Third, patients with COVID-19 who are being treated at RSUP dr. Wahidin Sudirohusodo, which is a referral hospital, is mostly a moderate-severe COVID-19 patient and is critical so that the effectiveness of convalescent plasma therapy in mild-moderate COVID-19 patients has not been carried out.

This publication is licensed under Creative Commons Attribution CC BY.

http://dx.doi.org/10.29322/IJSRP.11.12.2021.p12030

WwW.ijsrp.org 


\section{Conclusion}

From this study, it can be concluded that convalescent plasma therapy can be recommended, especially in moderate-grade COVID-19 patients. Further studies on a larger scale are needed regarding the effectiveness and timing of convalescent plasma administration in mild-moderate COVID-19 patients.

\section{References :}

AlQahtani M, Abdulrahman A, Almadani A, Alali SY, AlZamrooni AM, Hejab AH, et al. Randomized controlled trial of convalescent plasma therapy against standard therapy in patients with severe COVID-19 disease. Sci Reports. 2021;11:9927.

Alsharidah S, Ayed M, Ameen RM, Alhuraish F, Rouheldeen NA, Alshammari FR, et al. COVID-19 convalescent plasma treatment of moderate and severe cases of SARS-CoV-2 infection: a multicenter interventional study. Inter J Infect Dis. 2021;103:43946.

Brown BL, McCullough J. Treatment for emerging viruses: Convalescent plasma and COVID-19. Transfus Apheres Sci. 2020;59:1-5.

Duan K, Liu B, Li C, Zhang H, Yu T, Qu J, et al. Effectiveness of convalescent plasma therapy in severe COVID-19 patients. Proc Natl Acad Scie USA. 2020;117(17):9490-6.

Erkurt MA, Sarici A, Berber I, Kuku I, Kaya E, Ozgul M. Life-saving effect of convalescent plasma treatment in covid-19 disease: clinical trial from eastern Anatolia. Transfus Apheres Sci. 2020;59:1-3.

Gharbharan A, Jordans CCE, Geurtsvankessel C, den Hollander JG, Karim F, Mollema FPN, et al. Convalescent plasma for COVID19: a randomized clinical trial. Nature Com. 2021;12:3189

Maor Y, Cohen D, Paran N, Israely T, Ezra V, Axelrod O, et al. Compassionate use of convalescent plasma for treatment of moderate and severe pneumonia in COVID-19 patients and association with IgG antibody levels in donated plasma. E Clin Med. 2020;26:1-9.

Rejeki MS, Sarnadi N, Wihastuti R, Fazharyati V, Samin WY, Yudhaputri FA, et al. Convalescent plasma therapy in patients with moderate-to-severe COVID-19: A study from Indonesia for clinical research in low- and middle-income countries. E Clin Med. 2021;36:1-10

Sekine L, Arns B, Fabro BR, Cipollatt MM, Machado RRG, Durigon EL, et al. Convalescent plasma for COVID-19 in hospitalized patients: an open-label, randomised clinical trial. Europe Respir J. 2021;1-100.

Simonovich VA, Burgos Pratx LD, Scibona P, Beruto MV, Vallone MG, Vazquez C, et al. A randomized trial of convalescent plasma in Covid-19 severe pneumonia. N Engl J Med. 2021;384(7):619-29.

World Health Organization, 2014, Use of Convalescent Whole Blood or Plasma Collected from Patients Recovered from Ebola Virus Disease for Transfusion, as an Empirical Treatment during Outbreaks. Interim Guidance for National Health Authorities and Blood Transfusion Services .

World Health Organization, 2020, Infection prevention and control during health care when COVID-19 is suspected https://www.who.int/publications-detail/infection-preventionand- $\quad$ control-during-health-care-when-novel-coronavirus(ncov)- infection-is-suspected- 20200125

World Health Organization, 2020, WHO Director-General's remarks at the media briefing on 2019-nCov on 11 February 2020. Cited Feb 13rd 2020. Available on:https://www.who.int/dg/speeches/detail/who-director-generals-remarks-at-themediabriefing-on-2019-ncov-on-11-february- 2020. (Feb 12th 2020)

World Health Organization, 2020,. Laboratory testing for coronavirus disease 2019 (COVID-19) in suspected human cases. Interim guidance 2 March 2020

Ye M, Fu D, Ren Y, Wang F, Wang D, Zhang F, et al. Treatment with convalescent plasma for COVID-19 patients in Wuhan, China. J Med Virol. 2020;92:1890-901. 